\title{
Rapid Prototyping An Approach Beyond Manufacturing Technology
}

\author{
Dipl.-Inform. Jürgen Wagner ${ }^{1}$ \\ Dr.-Ing. Wilhelm Steger ${ }^{2}$
}

\begin{abstract}
This paper defines Rapid Prototyping as a new approach to reducing cycle times in product development, thus going beyond the view of Rapid Prototyping as a collective term only for the class of new, generative manufacturing technologies. In particular, the use of different categories of prototypes for the development of new products, and the organizational impact of Rapid Prototyping are shown to form a holistic framework for evolutionary product development. Depending on the extent to which the organizational principle of Rapid Prototyping is implemented, and depending on the individual utilization of specific prototypes, there may be a tremendous benefit in terms of the factors time, quality, and costs of the entire process. Furthermore, these benefits are not only due to the availability of physical prototypes. In all areas where virtual prototypes can provide answers to the core questions of a development process in the same manner as their physical counterparts, virtual prototypes perform at least as well (e.g., through reduced building time or cost reductions).

Before the influence of prototypes on the development process is discussed, there will be some general remarks on classes of prototypes and purposes they are used for. The need for a new organizational form is then derived from the requirements of designing, manufacturing, and evaluating the described prototypes, with cooperation and the respective enabling technologies turning out to be the essentials to the success of Rapid Prototyping in product development.
\end{abstract}

1. Fraunhofer Institute for Industrial Engineering and Organization (FhG IAO) Nobelstr. 12, D-70569 Stuttgart, Germany.

E-Mail: wagner@iao.fhg.de. WorldWideWeb: http://www.iao.fhg.de.

2. Fraunhofer Institute for Manufacturing Engineering and Automation (FhG IPA) Nobelstr. 12, D-70569 Stuttgart, Germany.

E-Mail: steger@qt.ipa.fhg.de. 


\section{Current Situation and Motivation}

International competition is characterized by an increasing dynamics of innovation. The gradually decreasing time of product marketing - in single cases, it is even shorter than the time of product development - requires enterprises to establish a growing number of new product developments and faster prototype development cycles in order to compete on the international market successfully. Thus, customer-oriented products which are tailored to the needs of particular target groups, as well as the early advertising of products gain more importance. The strategy of taylorization of work processes and the resulting, highly-specialized work distribution lead to deeply structured, hierarchical forms of organization, inhibiting not only fast reactions to changing customer requirements and short iteration cycles in product development, but also disabling innovation in enterprises. Consequently, such enterprises are not flexible enough to adapt to today's market's dynamics [Bull92].

Consequently, individual enterprises have to specialize their product lines while shortening iteration cycles during product development and maintaining a high innovation rate. Shorter product life cycles reduce monetary gains and lead to manufacturing sites moved off to countries offering cheap labour. As a direct result, the mutual interdependencies between and within companies become more and more significant and require the underlying organization to provide for a well-coordinated collaborative development process. As traditional tayloristic approaches quickly reach their limits due to the increasing complexity of the resulting processes, new methods for organizing the product development process are called for ([Bull93], [Bull94]).

However, not only the organization form has to be blamed for the decreasing success of many companies. There is also an insufficient support of the development process by information and production technology. Prototypes are still manufactured manually because of a missing CAD description, or worked out in a time-consuming milling or casting process, whereas a stereolithography apparatus could do it much quicker. Especially with regard to the increasing complexity of new and innovative products, prototypes play a key role for sharing ideas in multi-disciplinary teams. If this does not work, people fail to identify themselves with the product and become demotivated, which has tremendous influence on the quality not only of the work process, but also of the products.

All these problems mark simultaneously the goals and guidelines for a successful company. There is no doubt about the competitive edge of a faster time-to-market, but in order to fulfill all individual customer needs, increasing quality and decreasing costs of the products have to be considered as well.

Approaches like CIM (Computer-Integrated Manufacturing) or CSE (Concurrent/Simultaneous Engineering) deal with some of the described problems by introducing a high degree of information integration and well-defined information flows and processes. While such approaches are suitable for at least partially well-known tasks like the redesign of an existing 
product in order to improve functionality or quality, complex engineering tasks in the development of innovative products do not follow rules which can be modelled in the required, strict ways.

Innovation is closely related to creativity, i.e., a highly flexible work environment is needed to facilitate innovation and to eliminate constraining systems and rules, especially in the early phases of conception, where a formal product as such does not yet exist. It can be concluded that a integrative approach granting high flexibility is needed for product development [Bull94].

\section{Goals of Rapid Prototyping}

The Rapid Prototyping approach attempts to solve these problems by introducing into the product development process from the earliest stages

- an organizational form of distributed, autonomously responsible expert teams,

- techniques for the holistic integration of all knowledge along the product development process,

- methods for providing an integrated development and testing environment with both virtual and physical prototype manufacturing technologies, and

- techniques for networking all involved team members by means of intelligent cooperation and communication systems.

The key issue of Rapid Prototyping is the tight organizational and information-technological integration of the development team and all knowledge required to fulfill the development task, despite spatial separation, in order to shorten development iteration cycles, to decrease development cost by employing virtual as well as physical prototyping technologies, and to increase the utility of individual cycle steps through collaboration.

As a prerequisit for the right definition and design of a development process we first want to point out the use of prototypes, and how they can benefit in general the critical success factors of a company (i.e., time, costs, quality, flexibility).

\section{$3 \quad$ Prototypes in a Product Development Process}

\subsection{Classes of Prototypes}

A prototype is the result of the design and generation of one or more product characteristics which help the design team to test them against user requirements. According to this definition almost everthing can be a prototype. A classification is therefore recommended [Steg94] (see figure 1): 
- Design prototypes: They serve first and foremost for a design review under the consideration of optical, esthetical and ergonomical requirements, whereas mechanical aspects or accuracy are normally neglected.

- Geometrical prototypes: They are employed for testing accuracy, form and fit of the later series parts. Therefore the focus is on geometry and not on material aspects.

- Functional prototypes: They represent a set of features which allow the test of some funtional aspects. A functional prototype is usually a subsystem of a product.

- Technical prototypes: They cover all functional aspects of the part and can be used as such, but the manufacturing process is usually different from the one which will be later used in series production. The technical prototype may also consist of different material.

\begin{tabular}{|l|l|l|}
\hline $\begin{array}{c}\text { Design } \\
\text { prototypes }\end{array}$ & $\begin{array}{l}\text { Design review under the consideration } \\
\text { of optical, esthetical and ergonomical } \\
\text { requirements }\end{array}$ \\
\hline $\begin{array}{l}\text { Geometrical } \\
\text { prototypes }\end{array}$ & $\begin{array}{l}\text { Employed for testing accuracy, form } \\
\text { and fit of the later series parts. The } \\
\text { focus is on geometry and not on } \\
\text { material aspects. }\end{array}$ \\
\hline $\begin{array}{l}\text { Functional } \\
\text { prototypes } \\
\text { prototypes }\end{array}$ & $\begin{array}{l}\text { Functional aspects which are represented } \\
\text { as a set of features are reviewed (sub- } \\
\text { system of a product) }\end{array}$ \\
\hline
\end{tabular}

Figure 1: Classes of prototypes

However, not all prototypes have to be available in a classical sense as physical parts. For an example, take a CAD system which allows to visualize first design drafts, FEM software for the mathematical analysis of part structures, or a virtual reality machine that is envisaged to become an important design, evaluation and co-operation tool in the future. All these systems provide a non-physical, virtual prototyping, for all above mentioned classes of prototypes.

\subsection{Use of prototypes}

The benefit of prototypes emerges out of their use (see [Poll94], [UIri94]); four different cases can be identified (see figure 2). 


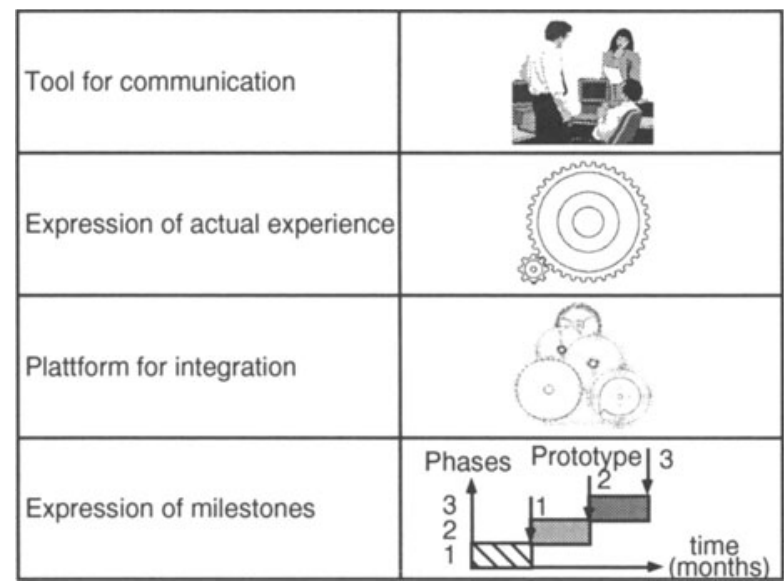

\section{Figure 2: $\quad$ Use of prototypes}

- Tool for communication:

Design is a process where a lot of people with different skills and views have to work together on the same product. For example a member of the top management has to explain his requirements to a designer, a mould maker and an expert for assembly tasks and vice versa. A prototype acts as a catalyst for such a discussion process.

- Expression of actual experience:

Maybe that the customer's requirements are very well defined at the beginning of a development process. Some uncertainty, however, may exist on how each requirement is fullfilled by a set of individual product features and how they can be put into reality. A prototype is therefore a tool for validation and verification, it expresses in each stage of the process the consolidated experiences of the customer and supplier.

- Platform for integration:

As we remember a functional prototype represents a subsystem of the final product. All subsystems have to be integrated and tested where constraints of assembly and co-operation have to be considered as vital. Such prototypes are well known as alpha- or beta-prototypes.

- Expression of mllestones:

The management and/or customers normally want to evaluate the progress of the development process at some stages. Only in case of a positive evaluation the project may go on. 


\subsection{Benefits of Prototypes for a Development Process}

Regarding the classes and the use of prototypes there can be indentified again four situations how a prototype can positively influence the development process (see [UIri94]). An interesting point is that only in the first case the speed of the prototyping process impacts directly the development process; in all other cases the mere availability of a prototype (instead of having none) is responsible for a shorter and - due to less iteration cycles - cheaper development.

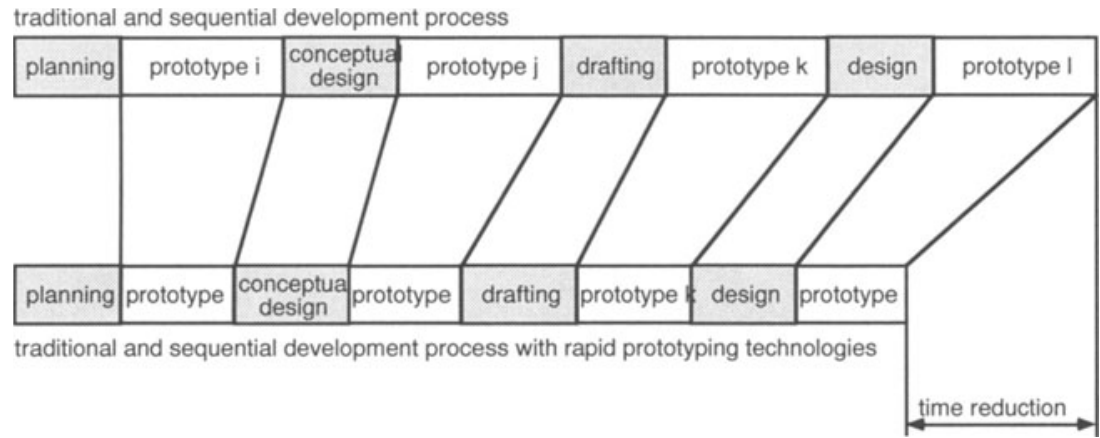

time (days)

Figure 3: Acceleration of the prototyping process

\subsubsection{Acceleration of the prototyping process}

By virtual prototyping prototypes can be made more quickly then with traditional physical methods (see figure 3). Yet the benefit depends on the complexity of the prototype and the set of requirements which should be evaluated. Furthermore there is a large number of prototypes in industry which encorporate electrical or hybrid (both mechanical and electrical) issues. Virtual prototyping, however, concentrates more on mechanical characteristics (geometry, esthetics, stress, fatigue, etc.) and can - at least within a short-term view - only apply in a phase of the development process which addresses these issues.

\subsubsection{A prototype influences later development phases}

In this case the prototype acts as a communication tool within the team and improves the decision finding. For example tool design can be done more quickly if a $3 \mathrm{~d}$ description of the part instead of a set of $2 \mathrm{~d}$ drawings - is available (see figure 4) or a complex physical prototype may be no longer necessary because a computer simulation provides the desired results (e.g., crash behaviour simulation). 


\begin{tabular}{|c|c|c|c|c|}
\hline part design & \multicolumn{3}{|c|}{ tool design } & tool production \\
\hline part design & prototype & tool design & tool production & \\
\hline \multicolumn{4}{|c|}{ tool production with prototypes } & time reduction \\
\hline
\end{tabular}

time (days)

Figure 4: Influence of a prototype on later phases

\subsubsection{A prototype improves the success rate of a development process}

Especially an early prototype allows a quick verification of the assumptions of the product development process. The reliabilty of these information, which serves as input to the next development phase, increases and also the probability of cost consuming changes in a later stage decreases (see figure 5). The costs of a prototype, however, have to be compared with those of a product change. Therefore prototyping is only recommended for risky and expensive products.

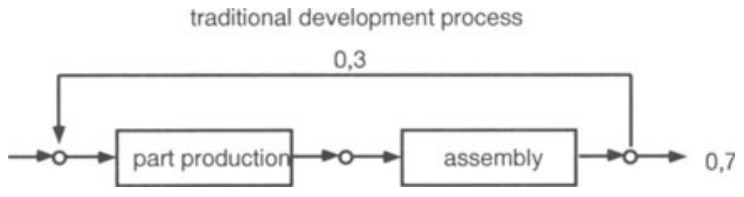

re-organised development process with prototypes

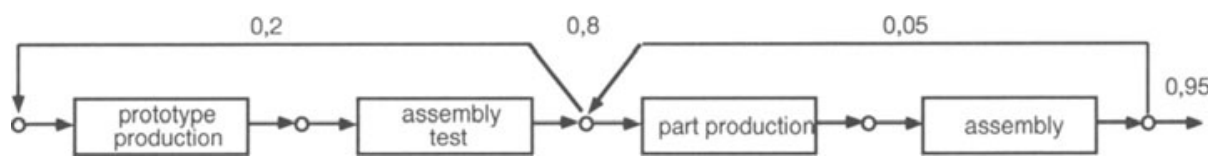

Figure 5: Increase of the success rate of a development process 


\subsubsection{Prototypes influence the sequence of the development phases}

The conventional sequence of the design of an injection mould is explained in figure 6 . Tests of the mould can be done only after it has been almost finished. Final assembly and test are influenced in line by a sequential process. A prototype (physical or virtual) helps to test the tool at an early stage and reduces both development time and uncertainty.
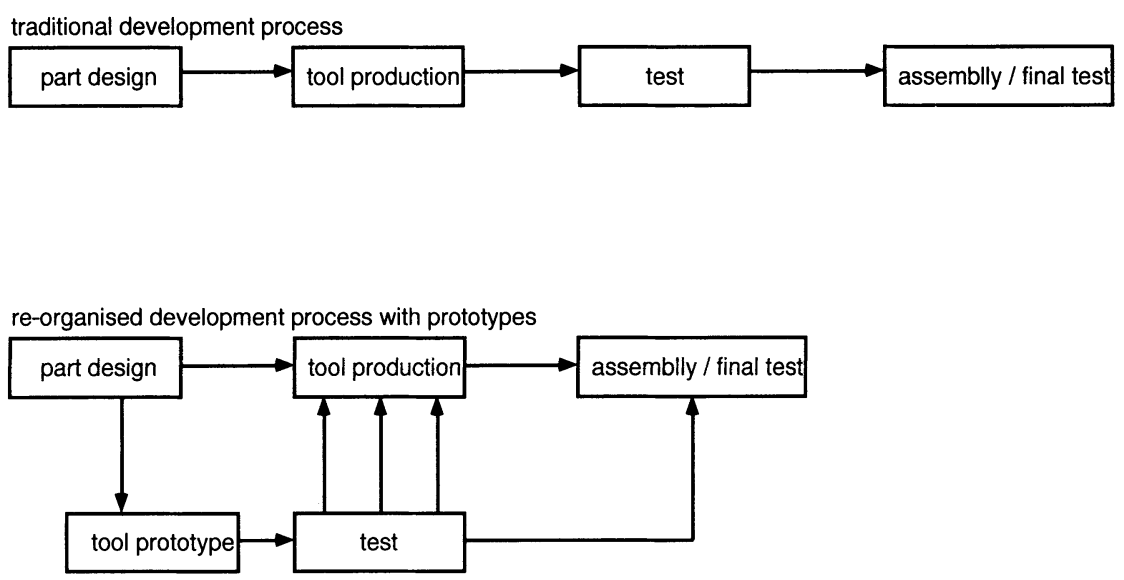

Figure 6: Reorganisation of the development process by prototypes

The following section will focus on the organizational framework required to gain the optimal utility from each of the described prototypes by applying Rapid Prototyping requirements to cooperation structures. In particular, a sketch of a future information-technological background directly facilitating this form of expert cooperation is described.

\section{$4 \quad$ Cooperation in Rapid Prototyping}

The inherently contradictory requirements of ideally maximizing quality while minimizing time and costs for both, development and production of prototypes or products, necessitate trade-offs to be made in most decisions regarding which prototypes may be designed and built in a particular stage of product development, in order to yield the desired results.

- Often, limitations on the development process are imposed by the availability of resources, e.g., supercomputing facilities. In such cases, simple prototypes may be used to investigate simple cases, while complex cases need to be studied in more detail, which requires more or a different type of resources. 
- In virtual prototyping, some features of a prototype design may not be demonstratable using entirely virtual prototypes, so a hybrid combination with a partially physical system may be required in order to combine the advantages of both approaches.

- In physical prototyping, the need to plan and to determine the utility of combinations of manufacturing technologies for a certain prototype arises as individual technologies implicitly define different physical properties and characteristics. For example, stereolithography may not be feasible if the thickness of separators lies below the manufacturable limits.

- Last, but not least, the managerial coordination of a development project requires trade-off decisions if several feasible alternatives exist but resources determine that only one or a few alternatives can be carried through. A similar situation can also be found in the evaluation phase of prototypes, where each aspect which was planned to investigate on a particular prototype, is examined. The gained results in the respective interation cycle step then need to be evaluated in a following exploitation step.

There is one common property in all of the above examples: two or more of the involved experts have to collaborate in order to obtain the desired results or decisions. The predominant reason for this is that whenever a decision involving even only partly opposing alternatives needs to be made, any single person will very likely be unable to make the best decision possible if the domain of alternatives is characterized by a significant amount of complexity. Therefore, the domain and decision complexity has to be reduced by letting individuals with specific know-how arrive at a partial interpretation of results or at a partial decision, and have these discussed in a team to check their mutual consistency or feasibility.

\subsection{Types of Complexity in Product Development}

Four distinct types of complexity can be identified in product development.

\subsubsection{Product complexity}

New products in mechanical and electronic engineering (e.g., trucks, cars, aircrafts, spacecrafts, ships, electronic devices supporting mechanical or thermodynamical systems) may involve thousands of parts. Even with a much smaller number of individual parts, fully understanding the interdependencies of functions of different components or assemblies usually requires thorough knowledge from a number of different engineering domains. With innovative products, this problem is even more significant, as there may be no long-standing knowhow as there is for conventional products. 


\subsubsection{Technological complexity}

The development of innovative products in fields of engineering implies the use of innovative technologies or the use of well-known technologies in new applications. Either way, the welldefined behaviour of new technologies or new combinations of manufacturing processes may not be well researched. In that case, product development also implies the first-time or refined development of stable and consistent characterizations of the processes in question.

\subsubsection{Knowledge complexity}

Given product and technological complexity, it is clear that several experts have to collaborate by providing expert knowledge on particular aspects of the entire product or prototype. The task of only partially integrating these different types of knowledge into a common pool in order to arrive at a common understanding and basis for investigations of possible solutions is one of the most difficult endeavors in multi-disciplinary work. The failure of traditional departmentalism with a plethora of formal interfaces, information-passing protocols, and release procedures stems from the lack of the common basis for discussing results.

\subsubsection{Organizational complexity}

Hierarchical development project structures oriented on the departmental structure of a company may imply a separation of work tasks that is not beneficial to reaching common goals by collaboration. The departmental or organizational separation of experts whose joint knowledge would be even more beneficial to the development process than each one's by itself causes artifical barriers where information reduced to mere data has to be passed. Clearly, welldefined results of work units are a prerequisite for a manageable project in terms of progress monitoring and controlling. However, this does not necessarily imply that those have to be the pieces of information passed between departments. They may as well be other, jointly achieved results which are defined based on the goals of the development project, not on the particular departmental structure of an organization.

\subsection{The Need for Cooperation}

The examples given above clearly indicate that a close collaboration between the different experts involved in achieving a common goal is required in order to facilitate

- an efficient exchange of information,

- the consideration of as many facettes of a problem as possible, and

- the time-effective accomplishment of a task.

Due to their inherent complexity, advanced development projects present problems of unpredictability (i.e., the product specification and work plan cannot be stated definitively at the outset) and concurrency (i.e., many interdependent subtasks are performed in parallel and may 
need to continuously exchange information). As a result, modifications of the original work plan or product specifications may be required within or after each iteration cycle. This type of work organization clearly cannot be accomodated by traditional management hierarchies [Amad93], but call for ad-hoc group decisions in conferences among the involved experts. This style of organization has been called "the networked organization“ [Malo91].

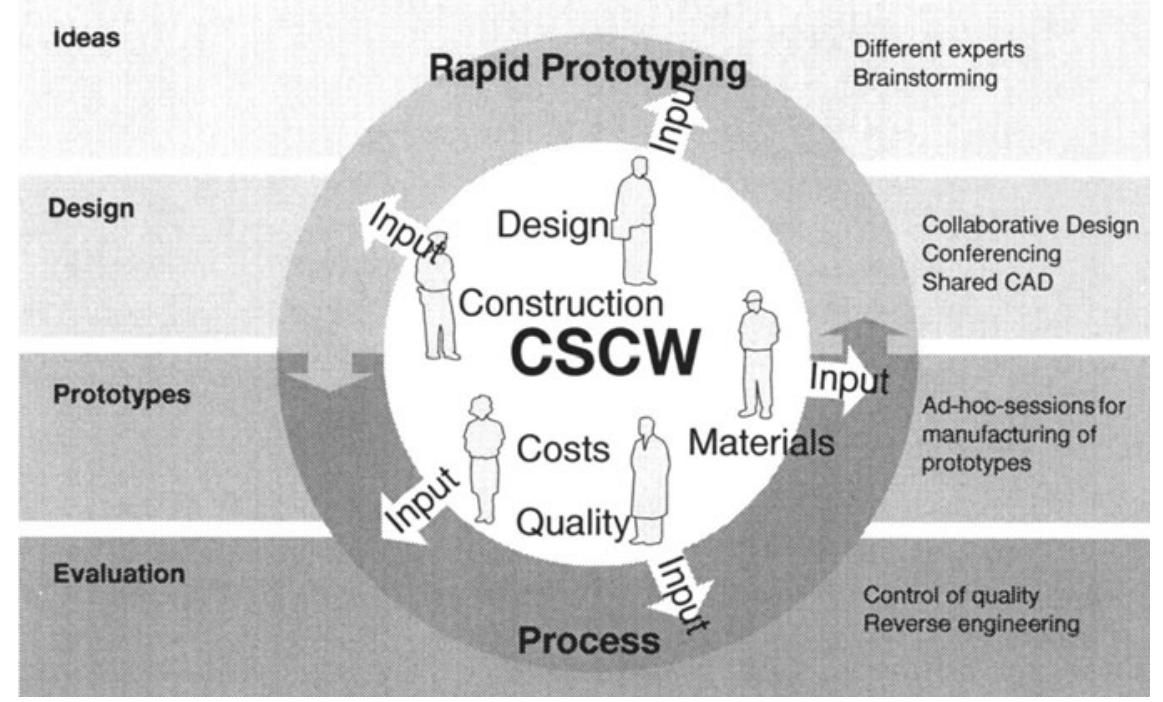

Figure 7: $\quad$ Cooperative work in Rapid Prototyping as a group process

Collaborative work is defined as the process of two or more individuals working together towards a common goal (e.g., a design, a prototype, an evaluation result, a managerial decision), with each individual contributing to the ultimate result. This organizational form requires all participating individuals to share a common stock of knowledge aiding in communicating essential information between them, and to reach a common understanding of subjects (figure 7). Consequently, expert training has to take a „T model“ into account: the horizontal bar symbolizes general knowledge needed to facilitate the understanding and participation in cross-domain, group decision processes, while the vertical bar represents detailed knowledge in an area of specialization. In order to contrast this type of experts to those found in tayloristic organizations, the term „generalist“ or „generalistic expert“ is used.

Computer-aided systems for various specialized fields of product development have been broadly employed during the last two decades. Despite the principal possibility of networking them together into an integrated environment, application programs (e.g., FEM, CAD) are single-user oriented, with only limited capabilities for data sharing and cooperation between users [Toye92]. As a result, they further solidify the work organization based on high specialization, 
and inhibit ad-hoc on-line cooperation. However, cooperation and communication are especially important in early phases of product design, where product data in the conventional sense only start to evolve as a result of team creativity and cooperation.

Rapid Prototyping is based on intensified collaboration. The most important prerequisites for effective cooperative engineering in distributed expert teams are

- the ability to utilize the flexibility gained from implementing the organizational form of teams of generalists, i.e., experts with a broad overview of not only their own field of specialization, but also that of others, and

- the support of the complex product engineering process by information and communication technology to achive an integration on the data, the application, and the team level.

The following section will elaborate the differences between the Rapid Prototyping approach and traditional development methods.

\subsection{Rapid Prototyping vs. Traditional Development Methods}

Rapid Prototyping can be viewed as an evolutionary process combining all technological, methodological, and organizational tasks from product conception to manufacturing into a single framework. In opposition to traditional approaches ([Bull92], [Köni93]) assigning specific prototypes to particular development phases, in Rapid Prototyping, the product of the development process evolves as a whole from early prototypes to the final product. This way, different dimensions of interest can be investigated in each iteration step, yielding information on particular aspects of the respective prototypes.

The rapidity of Rapid Prototying is achieved by the utilization of fast prototype manufacturing technologies (virtual prototypes, generative technologies), but also through the intensified cooperation of the development team. The constant interaction of team members guarantees dynamic, cross-functional links on demand, facilitating ad-hoc coordination and planning in direct response to questions and problems arising during the process of evolutionary product development. Clearly, this close interaction of developers also requires a seamless integration of tools.

Rapid Prototyping is orthogonal to sequential (conventional) or parallelizing (Concurrent Engineering) approaches suitable for development projects with mostly well-known structures. The strength of Rapid Prototyping shows in projects requiring the holistic, creative development of new, complex products, i.e., products where neither product nor project structures can be defined well in advance because they are subject to development within the project itself. However, a resonable combination of the three approaches proves to be the key to success: Rapid Prototyping may be used in the product definition and early design phases, including (in 
particular, virtual) prototype manufacturing and testing, whereas sequential and Concurrent Engineering methods may be employed in the subsequent refinement and optimization phases of the then well-defined product.

\subsection{IT Support for Rapid Prototyping}

In analogy to the different areas of complexity identified in a product development process, information and communication technology support must be granted to the development team.

\subsubsection{Product Design and Evaluation}

Major sources of product complexity are the number of components involved and the properties of functional or geometrical interdependencies between components. A Rapid Prototyping environment must accomodate this by integrating aids for appropriately organizing and intelligently retrieving these pieces of information. For retrieval, the individual needs of different experts have to be taken into account, i.e., it is essential that design and evaluation tasks may be performed cooperatively while allowing for individual views on commonly manipulated data. The acceptance and adequacy of a computer-based system significantly depends on its capability to provide each expert with the data representation or visualization he or she is used to work with.

\subsubsection{Prototype Manufacturing Technologies}

A correct supply of information is essential to all implementations of technology. The necessary information integration described for product design and evaluation also pertains to technological knowledge for manufacturing virtual and physical prototypes. A close cooperation of experts on candidate technologies for building a particular prototype is essential for a bestchoice selection among alternatives.

\subsubsection{Knowledge}

The integration of active knowledge facilitates not only a simplified exchange of information between experts from different domains, it also allows for an evolution of the knowlegde base from a first manual cooperation model to a more and more automated environment monitoring and controlling responsibilities and roles of development team members. This way, violations of imposed knowledge constraints (e.g., the modification of a diameter which also unknown to the designer affects the dimensioning of certain other components) can be handled by an emergency exit to a CSCW (Computer-Supported Cooperative Work) component of the Rapid Prototyping environment, letting the affected developers solve the problem, rather than trying to automate every decision. In fact, some decisions may require changes or relaxations to previously defined constraints, so an automatic treatment of these cases would not have been possible. However, by incrementally automating certain propagations of knowledge and decision guidelines, developers may delegate trivial aspects of their work to the IT support, concentrating on the creative task of designing the product. 


\subsubsection{Organization}

On the organizational level, there are three principal issues to be considered:

Project Management components need to accomodate the autonomous, spatially distributed work of team members and subteams. The project organization is likely to be flat, as opposed to deeply hierarchical structures in conventional organizations. Also, classical workflow approaches as provided in many Engineering Data Management Systems are not suitable to refine project tasks to a tool level, as they tend to impose a too rigid form on the product development process, incapable of accomocating rapid changes.

Computer-Supported Cooperative Work techniques need to be employed to support the synchronous or asynchronous interaction of humans through or with the aid of computer-based systems. The benefits of ad-hoc meetings for immediatly tackling problems, as opposed to filing engineering change requests or resorting to weekly meetings, have to be used in order to shorten communication paths between people. The "natural“ character of synchronous interactions with shared applications and audio (optional video) will also create a subjective feeling of togetherness within the team, despite spatial and temporal separation. In particular, once a „team spirit“ can be created, the readiness to accept the joint responsibility for the product development as a whole increases, thereby improving the motivation and quality of the work environment. An important role will play multi-media as an intuitively appealing, simple way of representing even complex information [Fran91].

Team Qualification is an important issue when an existing development team needs to be transitioned from the old departmental organization to a team organization. It may be necessary to offer special training courses in cooperation and team management, in order to facilitate effective team work. In contrast to group work in production, the social aspects and human factors involved in the collaboration of experts are widely unresearched.

\section{Conclusion}

This paper presented a view on Rapid Prototyping that includes all organizational, knowledge and technological aspects of product development.

Some key conclusions can be drawn:

- Rapid Prototyping as a process covering all stages of product development draws its rapidity in individual development cycles not only from technologies allowing engineers to quickly manufacture prototypes. It is vital for the success of a product development process to take as well Human Factors and organizational issues into account, and to specially encourage and support cooperative work. Cooperation is the key to effectively managing different types of complexity in product development. 
- The benefit of prototypes for development processes comes merely from the fact that we use prototypes without regard whether they are physical or virtual, but rather considering the degree to which new knowledge can be obtained from their evaluation. The organisation of the development process (i.e., in particular which prototype has to be used in which stage) has to be identified therefore as a critical point. There is, however, no generic organisation of a development process. It depends on the individual product.

- A flexible, fast, cost- and quality-effective development process combines a series of prototyping technologies. Virtual methods (CAD, simulation, FEM, Virtual Reality, etc.) and physical techniques (stereolithography, laser sintering, conversion tools, etc.) should both be applied dependent on the specific tasks in the process.

- The integration and best practice of many technologies requires a set of enabling technologies (e.g. expert systems, CSCW, adaptive information systems, databases and knowledge bases, product and process modelling). Both, the development of the enabling technologies and the prototyping technologies themselves have to be taken into account.

Many of the open research questions presented in this paper will be investigated within the Special Research Field (Sonderforschungsbereich) SFB 374 - Development and Testing of Innovative Products (Rapid Prototyping) - at the University of Stuttgart, which is partially funded by the German National Research Foundation (Deutsche Forschungsgemeinschaft DFG).

\section{References}

[Amad93] Amadio, P.; Fassina, I.: Case Studies. In: Power, R.J.D. (ed.): Cooperation aming organizations: the potential of Computer Supported Cooperative Work“. SpringerVerlag, Heidelberg, 1993.

[Bul192] Bullinger, H.-J.: Triaden-Management - Produktentwicklung im Blickwinkel veränderter Strukturen. In: Bullinger, H.-J. (Hrsg.) 4. F\&E-Forum:

Offensivstrategien für die Produktentwicklung, gfmt-Verlags-KG, München 1992.

[Bul193] Bullinger, H.-J.; Fremerey, F.; Fuhrberg-Baumann, J.: Innovative Production Structures - Precondition for a Customer-Oriented Production Management. In: Orpana, V.; Lukka, A. (ed.): Production Research 1993. Proceedings of the 12th International Conference on Production Research,Lappeenranta, Finland, 16-20 August, 1993, Amsterdam, London, New York, Tokyo: Elsevier 1993, S. 15-39.

[Bul194] Bullinger, H.-J.: Rapid Prototyping - Schneller zu neuen Produkten. In: Proceedings Fertigungstechnisches Kolloquium (FTK) ‘94. Stuttgart, November 1994. 
[Köni93] König, W; Eversheim, W.; Celi, I; Nöken, S; Ullmann, C.: Rapid Prototyping Bedarf und Potentiale. In: VDI-Z, 8/1993, pp. 92-97.

[Pol194] Pollmann, W.: Prototyping at Daimler Benz: State of the Art and Future Requirements. In: Proceedings of the IMS International Conference on Rapid Product Development, Jan 31 - Feb 2,1994, Stuttgart, Fraunhofer-Institute for Manufacturing Engineering and Automation (IPA), Stuttgart, 1994, pp. $241 \mathrm{ff}$.

[Fran91] Francik, E.; Ehrlich Rudman, S.; Cooper, D.; Levine, S.: Putting Innovation to Work: Adoption Strategies for Multimedia Communication Systems. In: Communications of the ACM, Vol 34, No. 12, pp 53-63.

[Malo91] Malone, T.W.; Rockart, J.F.: Computers, networks, and the cooperation. In: Scientific American, 265(3), pp. 84-91.

[Steg94] Steger, W.: Generative Fertigungsverfahren - Gewinn für den Prototypenbau und den Entwicklungsprozeß. In: Proceedings CAT/Quality ‘94,17. - 20. Mai 1994, Stuttgarter Messe und Kongreßgesellschaft, Stuttgart, 1994, pp. 156-164

[Toye92] Toye, G. et al.: SHARE: A Methodology and Environment for Collaborative Product Development. In: Proceedings of the IEEE Infrastructure for Collaborative Enterprises (CDR-TR \#19930507).

[UIri94] Ulrich, K.: The Role of (Rapid) Prototyping in (Rapid) Product Development. In: Proceedings of the IMS International Conference on Rapid Product Development, Jan 31 - Feb 2,1994, Stuttgart, Fraunhofer-Institute for Manufacturing Engineering and Automation (IPA), Stuttgart, 1994, pp. 195-205 